\title{
Thermodynamics of losses in photovoltaic conversion
}

\author{
Tom Markvart ${ }^{\mathrm{a})}$ \\ School of Engineering Sciences, University of Southampton, Southampton SO17 1BJ, United Kingdom
}

(Received 2 June 2007; accepted 5 July 2007; published online 7 August 2007)

\begin{abstract}
This letter presents a thermodynamic analysis of losses in an ideal solar cell. It is shown that the maximum voltage - corresponding to the voltage produced by a hot-carrier solar cell-is equal to the energy of the incident solar photon multiplied by the appropriate Carnot factor. Voltage generated by the usual $p-n$ junction cell is lower on account of entropy generation through kinetic losses, photon cooling, and étendue expansion of the incident beam. Simple expressions can be obtained by an approximation where the energy and entropy changes are modeled by the corresponding expressions for a two-dimensional ideal photon gas. (C) 2007 American Institute of Physics. [DOI: 10.1063/1.2766857]
\end{abstract}

A solar cell transforms a photon beam at temperature $T_{s} \cong 6000 \mathrm{~K}$ into electrical energy while emitting photons at temperature $T_{o} \cong 300 \mathrm{~K}$ of the solar cell. The photon flux in the beam is given by the usual expression

$$
\dot{N}(T, \mathcal{E}, \mu)=\frac{2 \mathcal{E}}{c^{2}} \int_{(\nu)} \frac{\nu^{2}}{e^{(h \nu-\mu) / k_{B} T_{o}}-1} d \nu,
$$

where $\nu$ is the frequency, the integration is over the spectral region $(\nu)$ of luminescence emission of the cell, $\mu$ is the chemical potential (in general, nonzero for luminescent radiation), and $\mathcal{E}$ denotes the étendue

$$
\mathcal{E}=\iint_{(A, \omega)} \cos \theta d A d \omega,
$$

where $d A$ is an element of the cross sectional area of the beam, $d \omega$ is an element of the solid angle, and $\theta$ is the angle between the normal to $d A$ and the direction of the beam. We assume that the cell represents a perfect, optically thick, emitter of radiation-in other words, it has emissivity equal to unity.

If, furthermore, the only recombination processes in the cell are radiative, the detailed balance between the electron current in the external circuit and the absorbed and emitted photon flows gives the $I-V$ characteristic of the cell:

$$
q J_{N}(\text { in })=I+q J_{N}(\text { out }),
$$

where $q$ is the electron charge,

$$
\begin{aligned}
& J_{N}(\text { in })=\dot{N}\left(T_{s}, \mathcal{E}_{\text {in }}, \mu_{\text {in }}=0\right)-\dot{N}\left(T_{o}, \mathcal{E}_{\text {in }}, \mu_{\text {amb }}=0\right), \\
& J_{N}(\text { out })=\dot{N}\left(T_{o}, \mathcal{E}_{\text {out }}, \mu_{\text {out }}=q V\right)-\dot{N}\left(T_{o}, \mathcal{E}_{\text {out }}, \mu_{\text {amb }}=0\right),
\end{aligned}
$$

$V$ is the voltage and $I$ is the current produced by the solar cell. Equations (4) assume direct (beam) illumination of the cell $\left(\mu_{\text {in }}=0\right)$ and take into account the fact that, in addition to sunlight, the cell is exposed to an ambient photon flux at zero chemical potential $\left(\mu_{\mathrm{amb}}=0\right)$ with étendue $\mathcal{E}_{\text {out }}-\mathcal{E}_{\text {in }}$. We note that the étendue $\mathcal{E}_{\text {out }}$ of the emitted beam must be greater than the étendue of the incident beam $\mathcal{E}_{\text {in }}$ since, by microscopic reversibility, photons can exit along the same path along which they arrived.

\footnotetext{
a)Electronic mail: tm3@soton.ac.uk
}

For weak to moderate illumination when stimulated emission can be neglected, the cell characteristic [Eq. (3)] simplifies to the usual form ${ }^{1}$

$$
I=I_{\ell}-I_{o}(\text { out })\left(e^{q V / k_{B} T}-1\right),
$$

where $I_{\ell}=q J_{N}($ in $)$ and $I_{o}($ out $)=q \dot{N}\left(T_{o}, \mathcal{E}_{\text {out }}, \mu_{\mathrm{amb}}=0\right)$. It is also useful to define $I_{o}($ in $)=\dot{N}\left(T_{o}, \mathcal{E}_{\text {in }}, \mu_{\mathrm{amb}}=0\right)$.

This letter gives an alternative thermodynamic description to this, essentially kinetic, view of the conversion process, by considering the energy and entropy balance of photons transferred between the incident and emitted photon beams. If propagating in a clear and transparent medium, these beams are characterized by constant thermodynamic parameters and act as basis states (or reservoirs); the transfer of photons between them produces work (or electrical energy) and, in general, a certain amount of waste heat through irreversible entropy generation.

Consider a packet of photons traveling with a beam defined by its temperature, étendue, and chemical potential where photons at the front of the packet are separated from the rear by a time interval $\delta t$. The gain in energy $(u)$ and entropy $(s)$ for a photon added to this packet can be calculated from the number of photons $N=\dot{N} \delta t$, energy $U=\dot{U} \delta t$, and entropy $S=\dot{S} \delta t$ of the packet by

$$
\begin{aligned}
& u=(\partial \dot{U} / \partial \dot{N})_{\mathcal{E}, T}, \\
& s=(\partial \dot{S} / \partial \dot{N})_{\mathcal{E}, T},
\end{aligned}
$$

since $\delta t$ can be taken as constant. Note that the étendue here takes on the role of volume in classical thermodynamics.

We can now proceed by analogy with irreversible thermodynamics (see, for example, Ref. 2) using the beams in the transparent medium as states of local equilibrium. The energy and entropy balance of the solar cell can then be expressed in terms of the thermodynamic energy and entropy flows $J_{U}$ and $J_{S}$ carried by the absorbed and emitted photon beams:

$$
\begin{aligned}
& J_{U}(\text { in })=J_{U}(\text { out })+\dot{Q}+\dot{W}, \\
& J_{S}(\text { out })=J_{S}(\text { in })-\frac{\dot{Q}}{T_{o}}+\frac{d_{i} S}{d t},
\end{aligned}
$$

where 


$$
\begin{aligned}
& J_{U}=J_{N} u, \\
& J_{S}=J_{N} s .
\end{aligned}
$$

$\dot{Q}$ is the rate of heat emission by the cell into a heat reservoir at temperature $T_{o}, \dot{W}=V I$ is the generated power, and $d_{i} S / \mathrm{dt}$ is the rate of internal entropy generation.

Equations (7) and (8) give a complete thermodynamic description of the photovoltaic conversion processes. Defining the entropy production per incident photon by

$$
\sigma_{i}=\frac{1}{J_{N}(\text { in })} \frac{d_{i} S}{d t} .
$$

Equation (7) gives the balance per incident photon in the form

$$
T_{o} \sigma_{i}=\left(u_{\text {in }}-u_{\text {out }}\right)+T_{o}\left(s_{\text {out }}-s_{\text {in }}\right) .
$$

In the language of classical thermodynamics, the entropy generation per photon [Eq. (10)] is seen to be equal to the change of availability restricted to processes at constant volume (see, for example, Ref. 3). Since $\mu_{\text {out }}=u_{\text {out }}-T_{o} s_{\text {out }}=q V$, the voltage can be written as

$$
q V=u_{\text {in }}\left(1-\frac{T_{o}}{T_{S}}\right)-T_{o} \sigma_{i}
$$

In other words, the voltage produced by the solar cell is equal to the energy of an incident photon, converted to the electrostatic energy $q V$ with the Carnot efficiency, less irreversible losses.

It will be seen that Eq. (11) represents the thermodynamic analog of the current-voltage characteristic [Eq. (3)] of the photovoltaic converter. It is interesting to note that, if the incident photon beam has a nonzero chemical potential $\mu_{\text {in, }}$ Eq. (11) can be written in a form which resembles the expression for entropy generation in irreversible thermodynamics,

$$
\frac{d_{i} S}{d t}=\left(\frac{1}{T_{o}}-\frac{1}{T_{S}}\right) J_{U}(\text { in })-\left(\frac{\mu_{\text {out }}}{T_{o}}-\frac{\mu_{\text {in }}}{T_{S}}\right) J_{N}(\text { in }) .
$$

The analysis of conversion losses becomes particularly transparent for weak to moderate radiation intensities when the energy $u$ and entropy $s$ reduce to the corresponding expressions of a two-dimensional ideal gas. Replacing the chemical potential by photon flux as the independent variable,

$$
\begin{aligned}
& u(T, \mathcal{E}, \dot{N})=h \nu_{o}+k_{B} T, \\
& s(T, \mathcal{E}, \dot{N})=k_{B}\left\{-\ln \left(\frac{\dot{N}}{\mathcal{E} T \gamma}\right)+1\right\},
\end{aligned}
$$

where $\gamma=2 k_{B} \nu_{o}^{2} / h c^{2}$ and $\nu_{o}$ is the minimum frequency of photons emitted by the converter-equal, for example, to $E_{g} / h$ for a semiconductor with band gap $E_{g}$. Equations (12) and (13) are readily obtained by differentiating the convective energy $\dot{U}$ and entropy $\dot{S}$ flows according to Eq. (6) in the limit when $\dot{N}$ is small. It is not difficult to show that the limits of applicability of Eqs. (12) and (13) coincide with the validity of the Shockley solar cell equation [Eq. (5)].

The entropy generation per photon $\sigma_{i}$ can now be written as a sum of three terms, $\sigma_{c}, \sigma_{\mathrm{kin}}$, and $\sigma_{\text {exp }}$, corresponding to Downloaded 28 Aug 2007 to 152.78.179.235. Redistribution subject

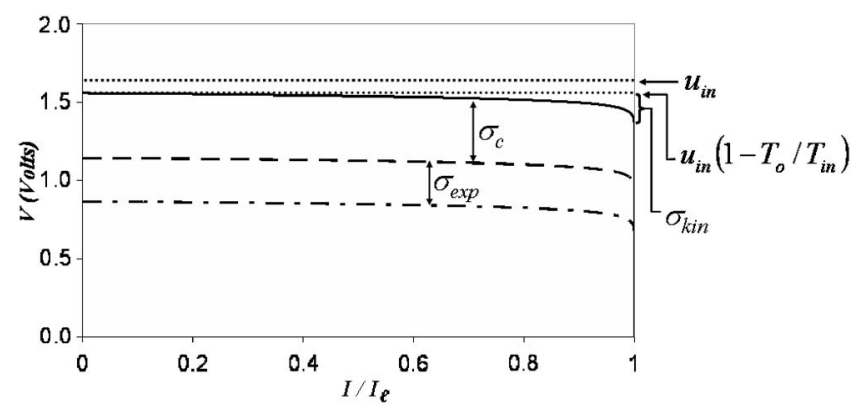

FIG. 1. Voltage-current characteristic for the different types of solar cells discussed in this letter. The dashed-dot line corresponds to 1 sun illumination, dashed line to sunlight with maximum concentration, and full line to hot-carrier solar cells. The threshold frequency $h \nu_{o}$ corresponds to crystalline silicon with $h v_{o}=1.12 \mathrm{eV}$.

the entropy changes in the individual steps $T_{s} \rightarrow T_{o}, \dot{N}_{\text {in }}$ $\rightarrow \dot{N}_{\text {out }}$, and $\mathcal{E}_{\text {in }} \rightarrow \mathcal{E}_{\text {out }}$ of the conversion process:

$$
\begin{aligned}
\sigma_{c}= & \frac{1}{T_{o}}\left(u_{\text {in }}-u_{\text {out }}\right)-\left\{s\left(T_{S}, \mathcal{E}_{\text {in }}, \dot{N}_{\text {in }}\right)-s\left(T_{o}, \mathcal{E}_{\text {in }}, \dot{N}_{\text {in }}\right)\right\} \\
= & k_{B}\left(\frac{T_{S}}{T_{o}}-1\right)-k_{B} \ln \left(\frac{T_{S}}{T_{o}}\right), \\
\sigma_{\text {kin }} & =s\left(T_{o}, \mathcal{E}_{\text {in }}, \dot{N}_{\text {out }}\right)-s\left(T_{o}, \mathcal{E}_{\text {in }}, \dot{N}_{\text {in }}\right) \\
& =k_{B} \ln \left(\frac{I_{\ell}+I_{o}(\text { in })}{I_{\ell}+I_{o}(\text { out })-I}\right) \cong k_{B} \ln \left(\frac{I_{\ell}}{I_{\ell}-I}\right), \\
\sigma_{\text {exp }} & =s\left(T_{o}, \mathcal{E}_{\text {out }}, \dot{N}_{\text {out }}\right)-s\left(T_{o}, \mathcal{E}_{\text {in }}, \dot{N}_{\text {out }}\right)=k_{B} \ln \left(\frac{\mathcal{E}_{\text {out }}}{\mathcal{E}_{\text {in }}}\right) .
\end{aligned}
$$

An additional term of the form $\sigma_{\text {nrad }}=-k_{B} \ln (\mathrm{QE})$, where $\mathrm{QE}$ is the quantum efficiency of the solar cell, can be included in $\sigma_{i}$ to represent nonradiative losses. ${ }^{4}$ It is easy to verify that all the terms that contribute to $\sigma_{i}$ are positive or zero, in accord with the second law of thermodynamics. Furthermore, the substitution of Eqs. (14)-(16) and (12) into Eq. (11) yields the Shockley solar cell equation, with the well known approximation ${ }^{5}$ for the open circuit voltage of a conventional solar cell. The resulting $I-V$ characteristic is shown in Fig. 1 as a function of voltage $V$.

The kinetic entropy term $\sigma_{\text {kin }}$ is the entropy generation on account of current extraction from the cell, and is nonzero as long as the cell produces finite current $I$. Clearly, this entropy generation term results from a finite "turnover rate" of the photovoltaic "engine," and is an unavoidable part of the conversion process.

The term $\sigma_{\text {exp }}$ is the entropy generation due to an isothermal expansion of the étendue if the étendue of the emitted beam is greater than the étendue of the incident beam (the related topic of angularly selective solar cells has been recently discussed by Badescu ${ }^{6}$ ). The corresponding energy $T_{o} \sigma_{\text {exp }}$ manifests itself as an increase of voltage produced by the solar cell upon concentration of sunlight. This term vanishes if the étendues of the incident and emitted beam are equal-achieved, for example, by focusing the incident sunlight to maximum concentration or by restricting the aperture of the emitted beam.

to AIP license or copyright, see http://apl.aip.org/apl/copyright.jsp 
The entropy term $\sigma_{c}$ which describes irreversible cooling of the beam from temperature $T_{S}$ to $T_{o}$ at constant étendue and constant photon flux represents one of the two fundamental losses in the Shockley-Queisser theory of ideal solar cell efficiency. ${ }^{1}$ There has been much discussion recently whether or how this irreversible entropy generation can be eliminated in a "hot-carrier" solar cell. ${ }^{7}$ The present thermodynamic analysis indicates that there is no fundamental reason why entropy generation $\sigma_{c}$ cannot be eliminated. Such hot-carrier device would make use of the difference between the energies $u\left(T_{S}\right)$ and $u\left(T_{o}\right)$ of the absorbed and emitted photons which results in free-carrier heating upon photogeneration of electron-hole pairs in the solar cell. A reversible conversion of this heat into useful work can be represented as an additional stage in the conversion process, and the work per photon can be added to the open circuit voltage at the terminals of the solar cell.

The Carnot cycle which represents this process absorbs heat by the absorption of high-temperature photons, and rejects heat through the emission of low-temperature photons together with an additional low-temperature heat term $T_{o} s_{\text {in }}$ $=T_{o} u_{\text {in }} / T_{s}$ to conserve entropy in the cycle. A relation appropriate for heat transfer is therefore used rather than the analogous relation for radiative transfer. This is reminiscent to the arguments leading to the Jetter and Petela-Press-Landsberg efficiencies for conversion of blackbody radiation into work. $^{8}$

If converted into electrostatic energy, work produced in the cycle increases the voltage to $\left(\mu_{\text {out }}+T_{o} \sigma_{c}\right) / q$, where $\mu_{\text {out }}$ is the chemical potential of the emitted photons, as before. As a corollary, we obtain that photons are emitted with a chemical potential which is always lower than the cell band gap $h \nu_{o}$. The resulting increase in efficiency can easily be calculated and is shown in Fig. 2.

The proposed hot-carrier enhancement of open circuit voltage differs from the hot-carrier cells proposed in Ref. 9 where carriers are extracted from the cell through selective contacts. The present method can also be contrasted with other (essentially kinetic) mechanisms for efficient utilization of the above band gap photon energy such as impact

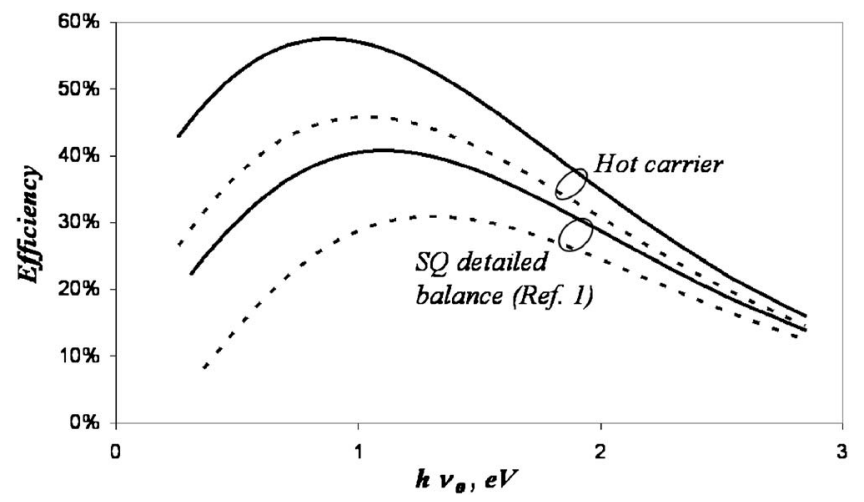

FIG. 2. Efficiency as a function of photon threshold energy $h \nu_{o}$. The dashed line corresponds to 1 sun illumination and full lines to sunlight with maximum concentration.

ionization enhancement ${ }^{10}$ or photon downconversion ${ }^{11,12}$ which seek to increase the current generated by the solar cell.

The author is indebted to Peter Landsberg for many stimulating discussions on the subject of this letter.

${ }^{1}$ W. Shockley and H. J. Queisser, J. Appl. Phys. 32, 510 (1961).

${ }^{2}$ S. R. deGroot and P. Mazur, Non-equilibrium Thermodynamics (Dover, New York, 1984),

${ }^{3}$ A. B. Pippard, The Elements of Classical Thermodynamics (Cambridge University Press, Cambridge, 1964),

${ }^{4}$ T. Kirchartz, U. Rau, M. Kurth, J. Mattheis, and J. H. Werner, Thin Solid Films 515, 6238 (2007).

${ }^{5}$ W. Ruppel and P. Würfel, IEEE Trans. Electron Devices ED-27, 877 (1980).

${ }^{6}$ V. Badescu, J. Phys. D 38, 2166 (2005).

${ }^{7}$ M. A. Green, Third Generation Photovoltaics: Ultra-High Efficiency at Low Cost (Springer, Berlin, 2003).

${ }^{8}$ See, for example, P. T. Landsberg, J. Appl. Phys. 54, 2841 (1983).

${ }^{9}$ R. T. Ross and A. J. Nozik, J. Appl. Phys. 53, 3813 (1982).

${ }^{10}$ S. Kolodinski, J. H. Werner, T. Wittchen, and H. J. Queisser, Appl. Phys. Lett. 63, 2406 (1993).

${ }^{11}$ T. Trupke, M. A. Green, and P. Würfel, J. Appl. Phys. 92, 1668 (2002).

${ }^{12}$ T. Markvart and P. T. Landsberg, Proceedings of the Third World Conference on Photovoltaic Solar Energy Conversion, Osaka, Japan, 2003, p. 289. 